\title{
Memory's reflection of learned information value increases across development
}

\author{
Kate Nussenbaum, Euan Prentis, Catherine A. Hartley \\ New York University \\ * correspondence to cate@nyu.edu
}

Citation: Nussenbaum, K., Prentis, E., \& Hartley, C.A. (2020). Memory's reflection of learned information value increases across development. Journal of Experimental Psychology: General. Advance online publication. doi: 10.1037/xge0000753 


\begin{abstract}
Prioritizing memory for the information most likely to be useful in the future is critical to learning effectively in our complex world. Previous work has revealed that the ability to strategically encode high-value information may improve gradually over development, as the systems supporting cognitive control processes mature. However, studies of value-directed memory have relied on explicit cues that signal the importance of information, which are rarely present in realworld contexts. Here, we examined whether individuals across age groups could learn the relative frequency of items in their environment and prioritize memory for information associated with higher frequency items, which would ultimately enable them to earn more reward. We found that from childhood to early adulthood, individuals gained the ability to dynamically adjust memory based on the statistics of the environment (Experiment 1). In the absence of any relation between item frequency and the reward that could be earned for encoding related information, the increased exposure to higher frequency items did not facilitate associative memory (Experiment 2). Taken together, results from our two experiments suggest that the use of past experience to prioritize memory for high-value information strengthens with increasing age and is supported by the developing ability to derive explicit knowledge of the structure of the environment from experience.
\end{abstract}




\section{LEARNED VALUE FACILITATES MEMORY OVER DEVELOPMENT}

\section{Introduction}

We encounter far more new information each day than we have the capacity to remember. This presents a problem: How do we selectively remember information we are most likely to need in the future? Selecting useful information for memory may be particularly important earlier in development. Relative to adults, children and adolescents encounter more novel information as they explore changing contexts, while also drawing on a less extensive knowledge base to support the integration of these new observations into rich, structural frameworks in memory (Bransford \& Johnson, 1972; Brod, Werkle-Bergner, \& Shing, 2013). Over the course of development, individuals get better at prioritizing memory for information they are explicitly told will be valuable to recall in the future (Castel et al., 2011; Hanten et al., 2007; Shohamy \& Adcock, 2010; Wittmann et al., 2005). In the real world, however, explicit signals of information value are often absent - it is impossible for parents or teachers to convey the relative importance of all the information that children encounter as they navigate dynamic environments.

In the absence of explicit cues, individuals may rely on various salience signals or prior schematic knowledge to prioritize memory. For example, individuals demonstrate enhanced memory for stimuli that elicit strong emotions (Adelman \& Estes, 2013; Schlüter, Hackländer, \& Bermeitinger, 2019), information that they are curious about (Fandakova \& Gruber, 2019; Kang et al., 2009; Marvin \& Shohamy, 2016; McGillivray, Murayama, \& Castel, 2015), novel items (Tulving \& Kroll, 1995), and schema-congruent information that can be easily integrated into preexisting knowledge frameworks (Brod \& Shing, 2019; Schlichting \& Preston, 2015). In some contexts, these memory biases may promote encoding of high-value information — remembering the face of a threatening person who provoked a strong emotional response could help one avoid a dangerous situation, and remembering the answer to a nagging trivia question could increase one's cocktail party capital. But in many cases, it is also useful to remember information that is not emotional, unusual, or embedded in a rich framework of prior knowledge, like the name of a new co-worker or the price of a loaf of bread across different grocery stores. Fortunately, the statistical structure of the environment itself can convey the value of remembering information ( $\mathrm{J}$. R. Anderson \& Schooler, 1991; R. B. Anderson, Tweney, Rivardo, \& Duncan, 1997). As we explore and learn from our surroundings across development, we may be able to prioritize memory for valuable information by applying more flexible and generalizable encoding strategies that draw on our past experiences of our environments.

The frequency with which we have previously encountered a specific item may be one important signal of the value of remembering associated information, because an item's past frequency often predicts its future frequency (J. R. Anderson, 1991; J. R. Anderson \& Milson, 1989; J. R. Anderson \& Schooler, 1991). For example, in their analysis of child language data, Anderson and Schooler (1991) found that the probability that a child would hear a given word on a particular utterance was related to the number of times they heard it in the 100 previous utterances. Learning the meaning of words that have been more frequent in the past might then enable comprehension of a greater proportion of spoken language in the future. Similarly, in patterns of social contact, the frequency with which one has encountered someone in the past 


\section{LEARNED VALUE FACILITATES MEMORY OVER DEVELOPMENT}

predicts the frequency of encountering them in the future (Pachur, Schooler, \& Stevens, 2014). Remembering the name of a frequently seen new coworker might then be more socially advantageous than that of a random passerby. Moreover, an item's past frequency may not only convey information value by predicting its future frequency - it may also predict its likelihood of being remembered (J. R. Anderson \& Milson, 1989). In this way, memory itself may be adapted to the distribution of information inherent to real-world environments.

Though prioritizing memory for information associated with high-frequency items might be adaptive at many ages, it is unclear whether and how the ability to implement this strategy emerges across development. To use frequency as a cue to prioritize memory for useful information, individuals need to learn the statistical structure of their environments and then use that structure to control encoding processes.

The ability to learn the statistics of one's environment is observable during infancy (Saffran \& Kirkham, 2017) and remains robust across childhood and adulthood (Amso \& Davidow, 2012; Meulemans \& Van der Linden, 1998; Potter, Bryce, \& Hartley, 2016; Schlichting, Guarino, Schapiro, Turk-Browne, \& Preston, 2017). However, even when children demonstrate sensitivity to contextual regularities, they often cannot explicitly report them (Finn et al., 2016; Wilhelm et al., 2013). Relative to recognizing repeated items in the moment, the ability to integrate multiple experiences to derive explicit knowledge of item frequencies may follow a more protracted developmental time course. Because past studies have relied on explicit cues (e.g. icons indicating monetary rewards for remembering specific items), one open question is whether the transformation of implicit to explicit knowledge is required for learned information to function as a value cue (Adcock et al., 2006; Castel et al., 2011a; Murty, Tompary, Adcock, \& Davachi, 2017; Shohamy \& Adcock, 2010). If item frequencies must be explicitly represented to influence subsequent encoding, then prioritization of memory based on past experience may improve into adulthood, mirroring the development of the ability to form explicit representations of environmental structure.

Beyond the cognitive mechanisms involved in detecting and representing signals of information value, the ability to control encoding processes based on these value signals might also determine how value influences behavior. The presence of value cues could, for example, cause participants to enhance or suppress their attention to memoranda (Uncapher \& Rugg, 2009) or to rehearse selected information in working memory (Craik \& Watkins, 1973). These momentby-moment adjustments of encoding strategy may rely on metacognitive awareness of one's own memory capacity as well as cognitive control processes to effectively implement them (Castel et al., 2011). The ability to use explicit value cues to proactively modulate control improves across development (Davidow, Insel, \& Somerville, 2018). Relative to children, adults and older adolescents are better, for example, at selectively enhancing attention (Störmer, Eppinger, \& Li, 2014) and inhibitory control (Insel, Kastman, Glenn, \& Somerville, 2017) when the reward they can earn for doing so is high. If the use of learned value signals to influence encoding similarly relies on the flexible engagement of cognitive control processes, then memory prioritization may 


\section{LEARNED VALUE FACILITATES MEMORY OVER DEVELOPMENT}

improve over development even after accounting for age-related differences in learning about environmental regularities.

Here, we examined how the development of the ability to learn about the structure of one's environment, integrate and explicitly access those regularities, and use them as value signals to modulate memory may support prioritization for useful information in the absence of explicit value cues. To address these questions, we had participants between the ages of 7 and 25 complete a novel laboratory experiment. Participants first learned the frequency of different items in their environment. Then, they learned information associated with each item and were told they would have to retrieve the associated information for every item in their environment, earning one point for each correctly retrieved association. In this way, the frequency of each item indicated the value of remembering information associated with it, mirroring many naturalistic environments in which the number of previous encounters with a particular item predicts the frequency with which related information will be needed in the future. Finally, participants' memories for both the learned associations and the initial item frequencies were probed.

We expected that participants across our age range would demonstrate comparable learning of item frequency, but that the ability to explicitly report item frequency and to use this learned information to control memory would improve gradually over development.

\section{Method}

\section{Experiment 1}

Prior to data collection, we preregistered our study design and analysis plan (including specific models and inference criteria) on the Open Science Framework using the "Preregistration Challenge" template. Our preregistration, along with the task code, de-identified raw data and analysis code, can be accessed on the Open Science Framework: https://osf.io/h9ncs/.

Confirmatory analyses describe results of statistical tests that were specified in our preregistration. Exploratory analyses describe results of additional analyses that were not explicitly preregistered. Other deviations from the preregistered plan are noted explicitly.

Our study design was modified from that of a prior, unpublished study (see Pilot Study on the Open Science Framework: https://osf.io/2qp7b/ ).

Participants. Ninety participants between the ages of 7.11 and 25.62 years took part in this experiment. Eleven additional participants were tested but excluded from all analyses due to a technical failure that caused some data files to be overwritten $(n=1)$, disclosing a learning disorder after testing $(\mathrm{n}=1)$, and performing below-chance on the paired-associates task $(\mathrm{n}=9$; see 'Experimental Tasks' for details). Because we did not know the size of our hypothesized effect, we based our sample size on other, similar studies of the development of decision-making and memory across childhood and adolescence (Decker, Lourenco, Doll, \& Hartley, 2015; Decker, Otto, Daw, \& Hartley, 2016; Unger, Ackerman, Chatham, Amso, \& Badre, 2016). Participants had normal or corrected-to-normal vision and no history of diagnosed psychiatric or learning disorders. The final analyzed sample of 90 participants comprised $n=30$ children (mean age $=10.07$ years; range $=7.11-12.93$ years; 15 females), $n=30$ adolescents (mean age $=15.43$ years; range $=13.1$ 


\section{LEARNED VALUE FACILITATES MEMORY OVER DEVELOPMENT}

-17.59 years; 16 females), and $n=30$ adults (mean age $=21.86$ years; range $=18.12-25.63$ years; 15 females).

Participants were recruited via flyers around New York University, and from science fairs and events throughout New York City. Based on self- or parent-report, 34.4\% of participants were White, 28.9\% were Asian, 23.3\% were mixed race, 12.2\% were African American and 1.1\% were Native American. Additionally, 21.1\% of the sample identified as Hispanic. Children and adolescents who participated in the study came from households with annual incomes that ranged from less than $\$ 20,000$ to more than $\$ 500,000$. For a fuller demographic characterization of the developmental sample, see Participant Details in the supplementary material.

Research procedures were approved by New York University's Institutional Review Board. Adult participants provided written consent prior to participating in the study. Children and adolescents provided written assent, and their parents or guardians provided written consent on their behalf, prior to their participation in the study. All participants were compensated $\$ 20$ for the 80-minute session. Participants were told that they would receive an additional bonus payment that would be based on their performance in the task; in reality, all participants received an additional $\$ 5$ bonus payment.

Experimental Tasks. Participants completed two sets of three tasks (Figure 1). The structure of each set of tasks was identical, but their narratives and stimuli differed. In one set of tasks, participants were told that they had a collection of postcards they needed to mail. Each type of postcard in their collection required a different type of stamp. Prior to beginning each experimental task, all participants received identical written and oral instructions. The instructions were written so as to be comprehensible to 7-year-olds and provided detailed information about each part of the task, along with specific examples of the types of images they would see and responses they would need to make. Further, participants were continually asked if they had any clarifying questions. The full task instructions are available within the task code on the Open Science Framework.

In the first task, the frequency task, participants were told they had to sort through their postcards to learn how many of each type they had. They were told that they had more of some types of postcards relative to other types (e.g. they might have 5 postcards with the same, specific blue pattern but only 1 postcard with a specific red pattern). Participants were instructed to try to keep track of how many of each kind of postcard they had, because it would be useful to them later on.

Throughout the task, participants viewed 16 images of postcards. Eight of these images were presented once and 8 of the images were presented five times, such that participants completed 48 trials total. On each trial, a postcard appeared in the center of the screen for 2 seconds, followed by a black screen $(500 \mathrm{~ms})$. Participants were instructed to press the "up" arrow key on a standard keyboard whenever they saw a type of postcard that they had previously seen. The specific postcard assigned to each frequency condition ( 1 or 5) was counterbalanced across participants. The order of image presentation was randomized for each participant. 


\section{LEARNED VALUE FACILITATES MEMORY OVER DEVELOPMENT}

In the second task, the paired-associates task, participants were told that they would learn the correct stamp to put on each type of postcard. We reinforced the relation between item frequency and the value of encoding information through explicit instruction: Participants were told that in the subsequent task, they would have to stamp all of their postcards, earning 1 point for each postcard stamped correctly. However, regardless of the number of each type of postcard that they had (i.e. 1 or 5), participants saw each type of postcard with its corresponding stamp only once. Participants were instructed that they would earn more points if they focused on remembering the stamps that went on the types of postcards that they had the most of. On each trial, participants viewed one of the types of postcards from the frequency task next to an image of a unique stamp (5 s), followed by a black screen $(500 \mathrm{~ms})$. Participants viewed each type of postcard with its corresponding stamp once, for a total of 16 trials. To ensure task engagement, participants were instructed to press an arrow key corresponding to the side of the screen on which the stamp appeared. When they pressed the correct key, a thin, grey box appeared around the image on the selected side for the remainder of the trial. The stamp-postcard pairs, order of the trials, and side of the screen on which the stamp and postcard appeared were randomized for each participant. Following the paired-associates task, participants took a 5-minute break.

Finally, participants completed the memory test. In the first part of the memory test, participants viewed all 16 unique postcards, one at time. When each postcard appeared, participants also saw all the stamps from the paired-associates task, as well as 4 novel stamps, arranged in a grid. Participants used the monitor's touch screen feature to press on the stamp that they thought belonged with the postcards, earning 1 point for each postcard stamped correctly. Participants had unlimited time to make their selection. After participants pressed a stamp, a grey outline appeared around it for $50 \mathrm{~ms}$, after which the postcard disappeared (500 ms) and a new postcard appeared. No feedback was given until the end of the task. The order of the postcard and the location of each stamp in the grid was randomized for each participant.

After stamping all 16 unique postcards once, participants' memory for the postcards' original frequencies was then probed. Participants again saw all 16 unique postcards, one at a time this time with the numbers $1-10$ underneath them. Participants were instructed to press on the number that they believed matched the number of times they saw the card in the first task. The order of the postcards was randomized for each participant.

Finally, participants stamped all remaining postcards, such that they completed 32 additional memory test trials (i.e. they stamped each of the postcards in the 5-frequency condition four more times.) These trials were not included in any analyses, but their inclusion ensured that correctly encoding the stamps that belonged on the high-frequency postcards would be more valuable for participants despite each retrieval trial being worth 1 point. At the end of the memory test, participants saw a screen that displayed how many postcards they stamped correctly.

After completing the three tasks, participants were told that they were going to play a second set of similar games. The second set of tasks was identical to the first, except that the stimuli were changed from postcards and stamps to landscape pictures and picture frames. The order of the stimulus sets was counterbalanced across participants. 
A.

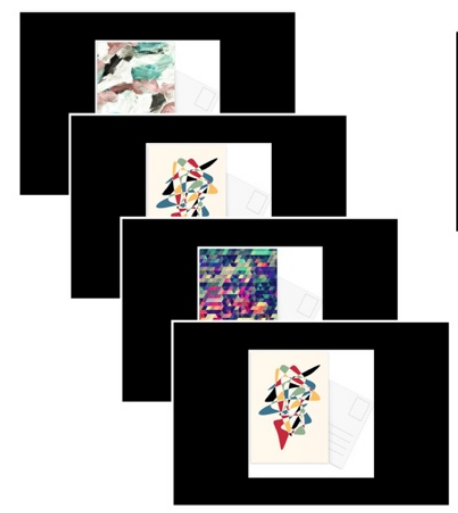

$2 \mathrm{~s}+500 \mathrm{~ms} \mathrm{ITI}$

B.

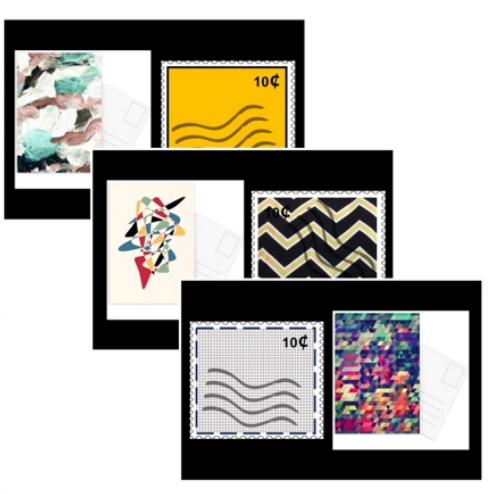

$5 \mathrm{~s}+500 \mathrm{~ms} \mid \mathrm{TI}$
C.

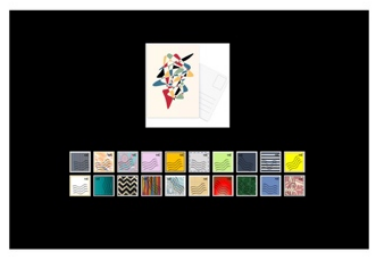

D.

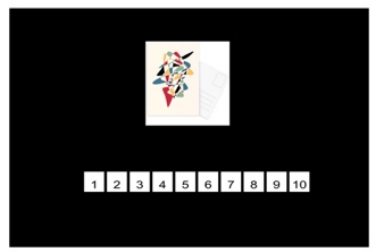

Figure 1. Task structure. Participants first learned the frequencies of each item (A) by viewing them in a continuous stream. They then were shown the information associated with each item (B). In the memory test, participants had to report the information associated with each item (C) as well as the item's original frequency (D).

At the end of the session, participants were administered the Vocabulary and Matrix Reasoning subtests of the Wechsler Abbreviated Scale of Intelligence (Wechsler, 2011). We followed the standard procedure to compute age-normed IQ scores for each participant based on their performance on these two sub-tests.

Analysis Approach. All data processing and statistical analyses were conducted in R version 3.4.2 (R Core Team, 2013). Mixed-effects models were run using the 'afex' package version 0.21-2 (Singmann et al., 2016). Numeric variables were z-scored across the entire data set prior to their inclusion in each model. To determine the random effects structures of our mixedeffects models, we began with the maximal model in order to minimize Type I error (Barr, Levy, Scheepers, \& Tily, 2013). We set the number of model iterations to one million and use the "bobyqa" optimizer. When the maximal model gave convergence errors or failed to converge in a reasonable time frame ( $\sim$ week), we removed correlations between random slopes and random intercepts, and finally, removed random slopes for interaction effects. In all cases, these slightly reduced models converged. For full details about the fixed- and random-effects structure of all models, see "Full Model Specification and Results" in the supplement.

To test the significance of the fixed effects in our models, we used likelihood ratio tests for logistic models and $\mathrm{F}$ tests with Kenward-Rogers approximations for degrees of freedom for linear models.

\section{Experiment 1 Results}




\section{LEARNED VALUE FACILITATES MEMORY OVER DEVELOPMENT}

Confirmatory analyses. Paired-associate task. First, we confirmed that all participants passed our simple attention check on each block of the paired-associates task. In this task, participants were instructed to press a button to indicate the side of the screen where the stamp (or the picture frame) appeared. A priori, we determined that we would exclude participants who responded incorrectly on more than four trials within a block. As described in the methods, nine additional participants ( $\mathrm{n}=3$ children; $\mathrm{n}=5$ adolescents; $\mathrm{n}=1$ adult) were tested but excluded from all analyses for failing this attention check on one or more blocks of the paired-associates task. The 90 participants who passed the attention check responded correctly on $94.7 \%$ (SD = $6.8 \%$ ) of trials.

Relation between age and IQ. We conducted a linear regression to determine the relation between age and IQ in our sample. There was a significant relation between age and IQ, $F(1,88)$ $=3.97, p=.049, \eta_{p}^{2}=.043$, indicating that the younger participants in our sample had higher IQ scores than older participants. As such, we included IQ as an interacting fixed effect in all of our models so that we could tease apart the influence of age from the influence of IQ on our measures of interest.

Learning of item frequency. Next, we examined how the accuracy and speed with which participants identified the repeating images in the frequency task changed as a function of age, block, and the number of times the item had appeared in the task. The data from three participants in Block 1 and two participants in Block 2 were excluded only from the frequency learning analyses because they responded to fewer than $10 \%$ of the repeating images, which suggests that they did not understand the task instructions. We did not preregister this exclusion because we did not anticipate this pattern of participant behavior. However, we wanted to ensure any effects we might observe of age on frequency learning were not driven by these participants. Further, though also not preregistered, we examined accuracy for the first appearance of each item separately from accuracy for all subsequent appearances of the items. When we examined our data visually, we saw that accuracy for repeated appearances of items seemed well-approximated by a linear model. But recognizing new images accurately was easier for participants than recognizing repeated images, leading to a discontinuity in accuracy between the first and second appearance of each item that could not be well-captured by a single linear model. We have included the results of our original, preregistered analyses in the supplement.

In general, participants were highly accurate in recognizing the new images, correctly withholding a response on $96.8 \%$ of trials $(S D=17.5 \%)$. To examine how participants' accuracy in recognizing the new images varied as a function of age and experiment block, we ran a logistic mixed-effects model. We observed a main effect of age on response accuracy, such that older participants were more accurate, $\chi^{2}(1)=12.85, p=.0003$. No other effects were significant ( $p$ s $>$ $.11)$.

Participants were also highly accurate in recognizing images as repeated, correctly responding on $92.6 \%$ of trials $(S D=26.1 \%)$. We examined how participants' accuracy in recognizing the repeated images varied across age, block, and the number of times each item had been presented (appearance count). We observed a main effect of appearance count on response 


\section{LEARNED VALUE FACILITATES MEMORY OVER DEVELOPMENT}

accuracy, $\chi^{2}(1)=65.2, p<.0001$, indicating that participants responded more accurately as the number of item repetitions increased. Accuracy also increased with age, $\chi^{2}(1)=10.26, p=.001$. Finally, we observed an age $\mathrm{x}$ IQ $\mathrm{x}$ block interaction effect, $\chi^{2}(1)=3.88, p=.05$. While both older participants and younger participants with higher IQs demonstrated equivalently high accuracy across both blocks, younger participants with lower IQs became more accurate in their responses from block 1 to block 2 .

To examine how participants' reaction times to the repeated items changed as a function of appearance count, age, and block, we ran a linear mixed-effects model. Our results indicated that participants were faster to respond to the repeated items as their appearance counts increased, $F(1,62.04)=111.55, p<.0001$. As with our accuracy data, we observed a main effect of age, $F(1$, $83.65)=9.97, p=.002$, as well as an age $\mathrm{x}$ appearance count interaction effect, $F(1,59.90)=4.21$, $p=.04$. Younger participants were slower to respond at the beginning of the task, but showed a greater increase in their speed of responding as the task progressed. We also observed a main effect of block, such that participants responded more slowly in the second block, $F(1,78.15)=4.88, p$ $=.03$. No other effects were significant $(p \mathrm{~s}>.08)$.

Taken together, our data from the frequency-learning phase of our experiment suggest that, though older participants responded slightly more accurately and with greater speed, participants across our age range were able to recognize both the new and repeated images with a high degree of accuracy. Additionally, participants became more accurate and faster as the item appearance count increased. The reaction time effect was particularly pronounced in younger participants, suggesting that their recognition of the repeated items may have continued to improve more sharply upon subsequent presentations of the images. Further, after completing one entire block of tasks and realizing they would be directly tested on the item frequencies, participants may have adjusted their strategy and slowed their responding to better learn the frequency information.

Explicit frequency reports. Next, we examined participants' explicit knowledge of the frequency of each item by examining the magnitude of participants' report error on each trial. To compute error, we took the absolute value of the difference between the true, underlying frequency condition ( 1 or 5 ) and subtracted it from the number of times that participants reported seeing the item $(1-10)$. In our preregistration, we specified that we would examine the raw frequency reports. However, we hypothesized that younger participants would perform less accurately, but not that they would be systematically report frequencies that were too low or too high. To test this hypothesis more directly, we deviated from our analysis plan and examined the report error magnitudes (the absolute values of the report errors), rather than the reports themselves. We have included our original preregistered analysis of frequency reports in our supplement.

We examined the influence of frequency condition, block, age, IQ, and their interactions on these report error magnitudes with a linear mixed-effects model. As we hypothesized, report error magnitudes decreased with age, $F(1,85.52)=12.67, p=.0006$ (Figure 2). There was no difference in report error magnitudes across frequency conditions, $F(1,93.94)=.01, p=.92$. No other effects were statistically significant $(p s>.05)$. 


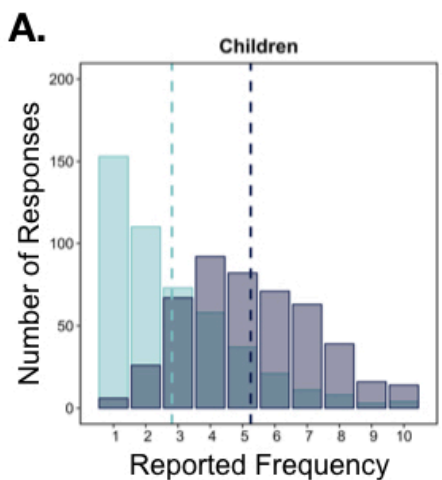

B.

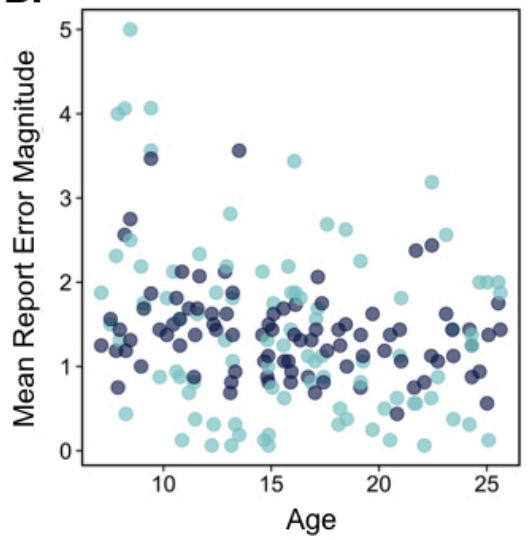

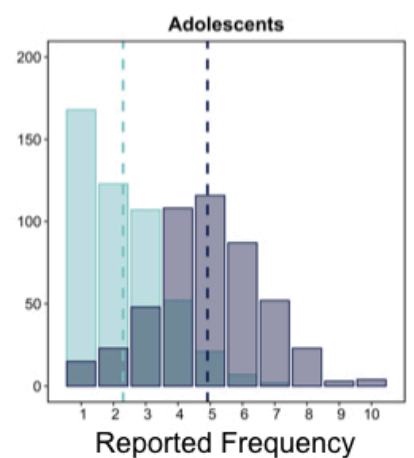

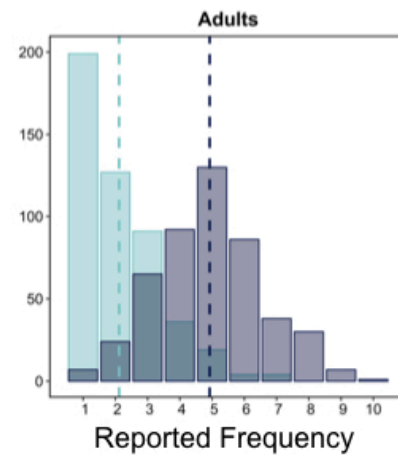

C.

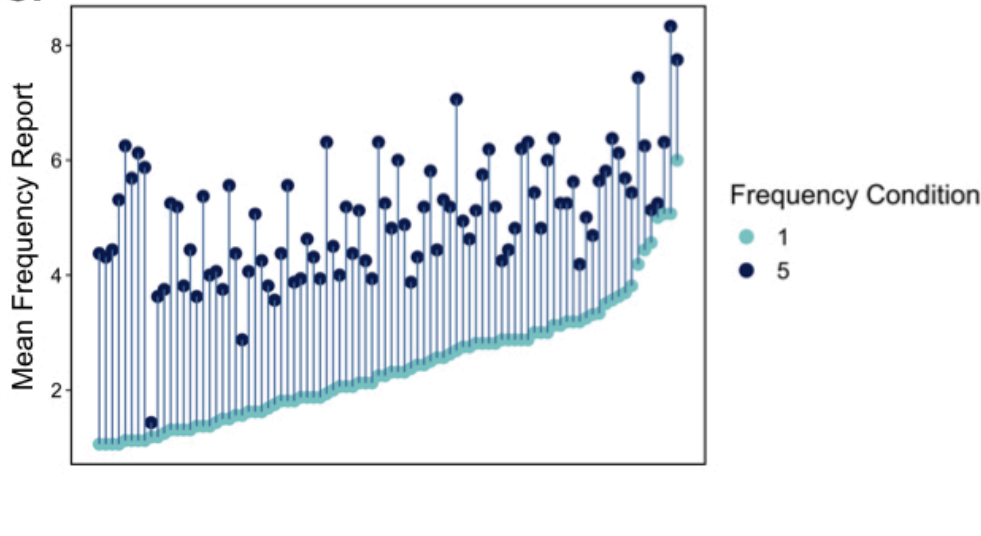

Figure 2. A) Participants across age groups successfully differentiated the items in the 1frequency condition (green) from those in the 5-frequency condition (gray), as indicated by the different distributions of explicit frequency reports. The dotted lines represent the average frequency report for each frequency condition. B) Average frequency report error magnitudes for each participant in each condition. There was no difference in report error magnitudes across frequency conditions. With increasing age, participants' reports became more accurate. C) All participants reported the items that appeared five times to have been more frequent than those that appeared once.

This result indicates that participants' ability to transform their on-line learning experience into explicit knowledge improved with developmental time. However, as Figure 2 shows, participants across our age range successfully differentiated low- from high-frequency items in their explicit reports.

Value-guided memory. After confirming successful learning and memory for item frequencies, we turned to our main question of interest: Beyond simply learning and reporting the frequencies of items in their environments, could participants across our age range use these learned frequencies to strategically prioritize high-value information in memory? To address this question, we computed participant's memory accuracy on each trial within the first part of the memory test, when participants had to identify the correct paired associate for each item for the first time. Trials were scored as accurate if participants selected the correct associate from the grid of 20 options and inaccurate if they selected any of the other 19 options, such that chance-level performance was $5 \%$. We examined how frequency condition, block, age, IQ, and their 
interactions influenced participants' memory accuracy with a logistic mixed-effects model. Older participants remembered more item pairs overall, $\chi^{2}(1)=30.33, p<.0001$ as did participants with higher IQs, $\chi^{2}(1)=5.59, p=.0009$. In line with our hypothesis, participants were significantly more accurate in remembering the paired-associates of the items in the 5-frequency condition relative to those of the items in the 1 -frequency condition, $\chi^{2}(1)=16.45, p<.0001$. Critically, this effect was qualified by an age $\mathrm{x}$ frequency condition interaction, $\chi^{2}(1)=5.59, p=.02$, such that the difference in memory performance across frequency conditions increased with increasing age (Figure 3).

We also observed an age $\mathrm{x}$ IQ interaction effect, $\chi^{2}(1)=8.82, p=.003$, which indicated that IQ influenced overall memory performance to a greater degree at younger ages. However, IQ did not interact with frequency condition, suggesting that while higher-IQ participants were better at remembering information, they were not necessarily better at remembering high-value information. Additionally, participants' overall memory performance improved across blocks, $\chi^{2}(1)=15.6, p<.0001$. Importantly, we did not observe a significant frequency condition $\mathrm{x}$ block interaction effect $\chi^{2}(1)=.85, p=.36$, indicating that participants may have learned general strategies to enhance encoding across the experiment, but that they did not selectively get better at remembering the high-value items with practice.
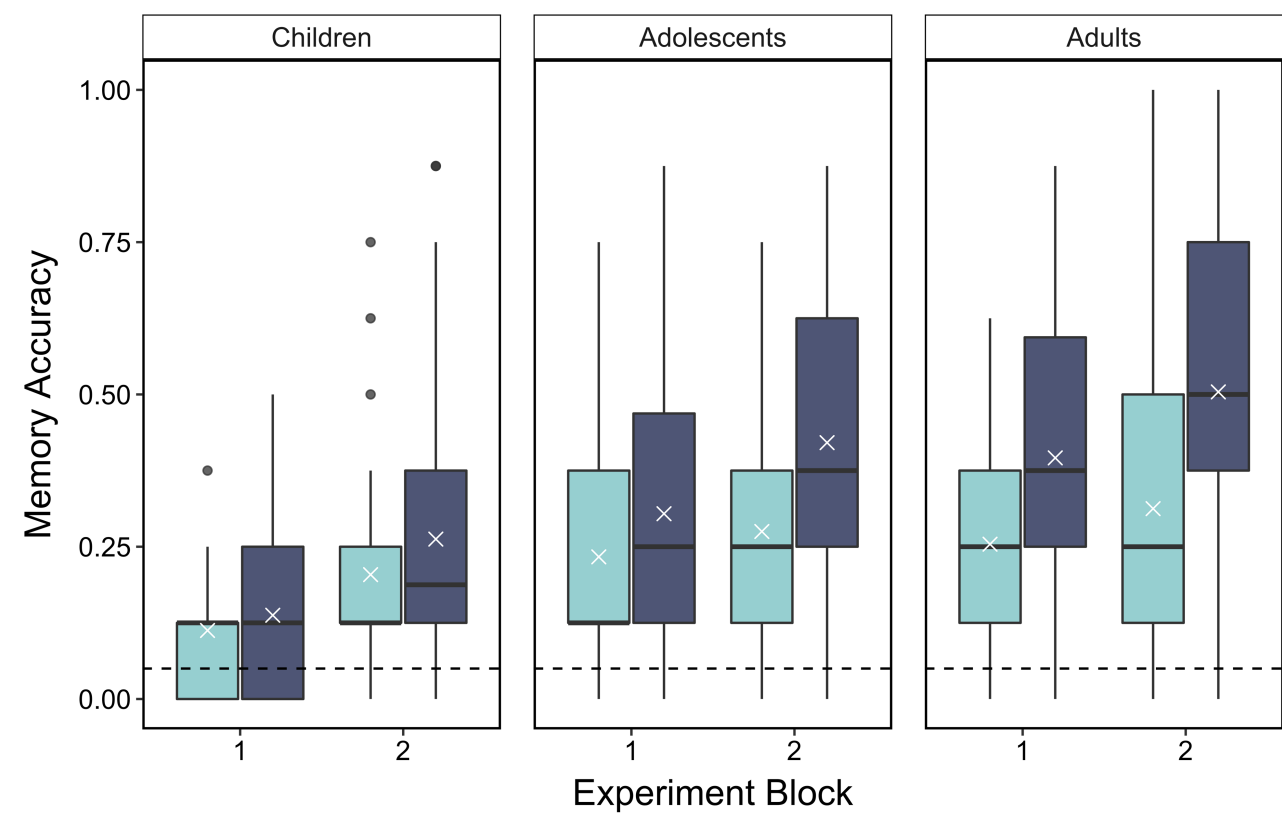

Frequency Condition

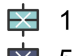

文 5

Figure 3. With increasing age, participants demonstrated a greater ability to prioritize memory for high-value information, as indicated by higher memory accuracy for information associated with items in the 5- relative to the 1 -frequency condition. White Xs indicate mean accuracy for each group. The horizontal black lines indicate median accuracy values. The lower and upper edges of the boxes indicate the first and third quartiles of the grouped data, and the vertical lines extend to the smallest value no further than 1.5 times the interquartile range. Black dots indicate data points outside those values. The dashed line indicates chance-level performance. 


\section{LEARNED VALUE FACILITATES MEMORY OVER DEVELOPMENT}

Relation between frequency reports and value-guided memory. Finally, we examined the relation between explicit knowledge of the structure of the environment and the influence of item frequency on memory. We hypothesized that participants who represented the high- and lowfrequency items as differing in value to the greatest extent would also show the greatest degree of memory selectivity for pairs involving high-frequency items. To test this relation, we computed two variables for each participant for each block: We computed a frequency distance index by subtracting participants' average frequency report for items in the 1-frequency condition from their average report for items in the 5-frequency condition and dividing this value by the standard deviation of all of their frequency reports. We also computed a memory benefit index by subtracting participants' average memory accuracy for the paired associates in the 1-frequency condition from their average memory accuracy for the paired-associates in the 5-frequency condition and dividing this value by their overall mean memory accuracy. We predicted that participants whose frequency reports for items in the 1- and 5-frequency conditions differed to the greatest extent would also demonstrate the greatest difference in their memory for the paired associates across the two frequency conditions. Additionally, we thought this relation might grow stronger with increasing age, with older individuals demonstrating greater use of learned value signals to adaptively adjust their control of encoding on a trial-by-trial basis.

To examine how the frequency distance index, age, and experiment block related to the memory benefit index, we ran a logistic mixed-effects model. Beyond these interacting fixed effects, our model additionally included IQ as an interacting fixed effect and a random intercept for each subject. As we hypothesized, we observed a significant effect of frequency distance on the memory benefit index, $F(1,135.89)=4.52, p=.04$, indicating that participants who remembered the frequencies as further apart also preferentially encoded the high-value information to a greater extent. However, contrary to our expectation, this relation was not influenced by age, $F(1,146.67)=.39, p=.54$. That said, since this analysis only had two data points for each subject, we may have been underpowered to detect age effects. Indeed, we did not observe a significant main effect of age on memory benefit index $(F(1,82.71)=.1 .58, p=.21)$, which is surprising given our previous results which indicated increasing prioritization of high-value items with increasing age.

Exploratory analyses. Item-level relation between frequency reports and memory. Our preregistered analysis examined how participants' ability to distinguish the two frequency levels in their explicit reports related to their ability to prioritize memory for high-value information. But our participant-level analysis does not reveal how beliefs about the frequency of a given item influenced how information associated with it is prioritized in memory. To better address this question, we ran an additional logistic mixed-effects model to examine how the individual frequency report for each item, along with block, age, and IQ influenced memory accuracy. As before, we observed better overall memory performance with increases in age, block, and IQ ( $p$ s $<.002$ ) (Figure 4), as well as an age x IQ interaction effect on memory $(p=.01)$ with IQ influencing overall memory accuracy to a greater extent at younger ages. Critically, we observed a main effect 
of frequency report, $\chi^{2}(1)=30.67, p<.0001$, such that participants demonstrated better memory for items they reported as more frequent. Mirroring our frequency condition results, we found that with increasing age, the influence of explicit frequency report on memory performance grew stronger, $\chi^{2}(1)=6.13, p=.01$. Finally, we also observed an age $\mathrm{x}$ IQ $\mathrm{x}$ frequency report interaction effect $\chi^{2}(1)=5.49, p=.02$, such that adolescents and adults with higher IQs demonstrated greater modulation of memory performance based on reported frequencies. This result suggests that individuals with the greatest capacity for cognitive control - older participants with higher IQs - may have been better at strategically adjusting memory encoding based on their knowledge of the structure of the environment. Together with our previous analysis that showed that children make more errors in reporting item frequencies, these results indicate that value-guided memory may depend both on the development of the ability to integrate multiple experiences to form explicit knowledge and the ability to use this explicit knowledge as a value signal during encoding.
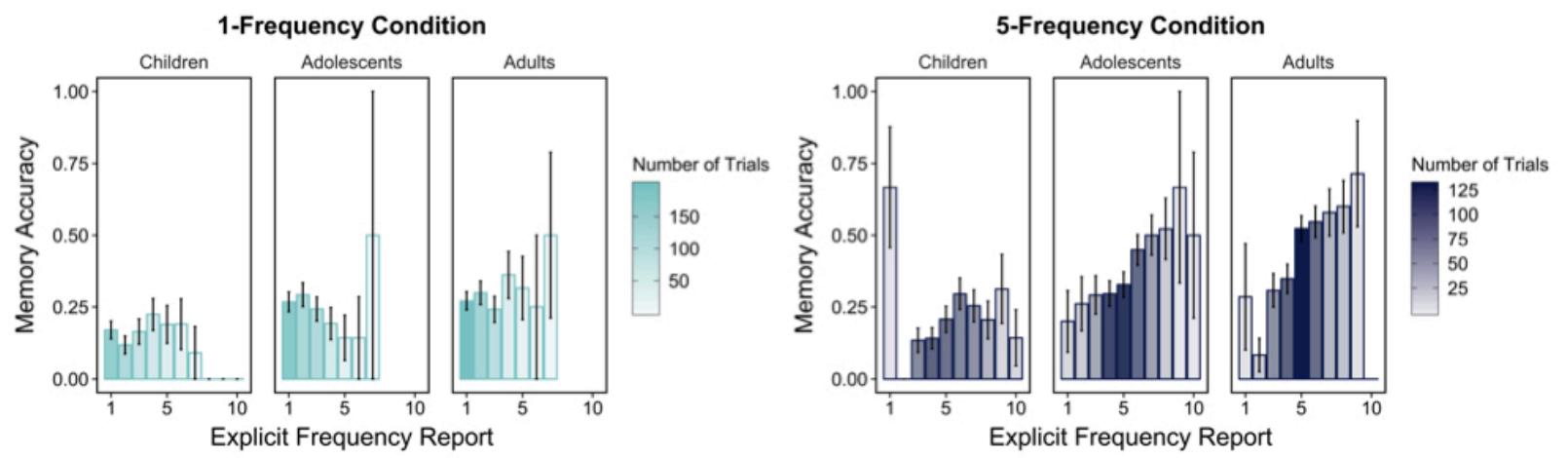

Figure 4. Participants demonstrated increased memory accuracy for items that they reported as being more frequent in both frequency conditions. This effect emerged with increasing age. The shading of the bars represents the number of trials on which participants provided that frequency report. Reports closer to the true underlying frequencies -1 and 5 - were more common.

While this analysis suggests that participants' beliefs about item frequency, reflected in explicit reports, influenced memory performance, these reports are inherently correlated with the true frequency of the item. To determine whether participants' explicit frequency reports explained memory performance above and beyond the underlying frequency condition, we compared our explicit frequency report memory model with our original model that examined the effect of frequency condition on memory. A likelihood ratio test revealed that the model with the explicit frequency reports (Bayesian information criteria $(B I C)=3240.9)$ fit the data significantly better than the model with frequency condition (BIC $\left.=3260.8), \chi^{2}(1)=19.901, p<.0001\right)$. This suggests that deriving explicit knowledge of the statistical structure of the environment directly contributes to memory prioritization above and beyond simply experiencing those statistics.

Relation between frequency report error and memory. Our prior analysis suggests that participants' beliefs about the structure of the environment influence memory prioritization even if those beliefs are incorrect. In other words, if participants believed that an item appeared more 


\section{LEARNED VALUE FACILITATES MEMORY OVER DEVELOPMENT}

times than it actually did, they tended to demonstrate enhanced memory for information related to that item. However, it may also be the case that participants had better memory for information related to items for which they were able to accurately report frequency. To test this possibility, we conducted a logistic mixed-effects model to examine how the frequency report error magnitude for each item, along with block, age, and IQ influenced memory accuracy. As before, we observed positive main effects of age, block, and IQ on memory performance $(p s<.001)$. We did not, however, observe an effect of frequency report error magnitude on memory, $\chi^{2}(1)=.57, p=.45$. We similarly did not observe a significant effect of frequency report error magnitude on memory when we included frequency condition as an interacting fixed effect in the model $(p=.40$; see Supplementary Analyses, Table S32). Thus the accuracy with which participants transformed their statistical learning experience into explicit representations of item frequencies did not directly influence memory performance on an item level. Taken together with our prior analysis, these results suggest that the magnitude of frequency reports had a greater influence on memory than the accuracy of frequency reports. In other words, participants tended to demonstrate better memory for low-value paired associates they believed were of high value relative to those they correctly identified as not being very valuable.

\section{Experiment 1 Discussion}

In Experiment 1, we examined how learning about environmental regularities may enable people to preferentially encode useful information. Specifically, we designed tasks to mirror the structure of many real-world environments in which an item's past frequency predicts how often one might encounter the need to remember information associated with it in the future (e.g. Anderson, 1989). We found that from middle childhood to early adulthood, individuals gained the ability to use the natural statistics of their environments to encode high-value information. We extended past research on value-guided memory by demonstrating that prior learning about the structure of the environment can serve as an effective value signal that facilitates subsequent memory.

In addition to age, individual differences in IQ also affected memory performance. Participants with higher IQs demonstrated better memory overall, and adolescents and adults with higher IQs demonstrated a greater influence of reported frequency on memory. These results are consistent with prior literature that suggests a relation between IQ and explicit memory (Reber, Walkenfeld, \& Hernstadt, 1991) and between IQ and attentional control (Kane \& Engle, 1991).

Older participants were not only better at prioritizing memory for high-value information, they were also more accurate than younger participants in recognizing repeated items in the first, frequency-learning task, and in transforming their experiential learning into explicit reports of item frequency. Older participants' lower frequency report error magnitudes may reflect developmental improvements in the ability to represent and access knowledge of learned contextual regularities (Finn et al., 2016; Wilhelm et al., 2013). Still, our findings stand in contrast to previous work on developmental change in frequency judgments, which has found only weak evidence for agerelated increases in frequency sensitivity (Hasher \& Zacks, 1984). In one study, first- and secondgrade children and young adults completed a task in which they saw a continuous stream of words 


\section{LEARNED VALUE FACILITATES MEMORY OVER DEVELOPMENT}

and, for each word presented, had to estimate the number of times they had seen it previously. Here, young adults' judgments showed a slightly higher correlation with the true word frequencies, though a second experiment revealed no age-group differences (Ellis, Palmer, \& Reeves, 1988). In another experiment children and adults who had to report item frequencies after viewing lists of items performed equally well, both when they were and were not forewarned of the upcoming frequency knowledge test (Hasher \& Chromiak, 1977). Our frequency-learning task, which involved more abstract images, may have been more challenging than the word lists used in prior work; the stimuli we used may have been more easily confusable with each other, such that developmental refinements in the specificity of memory representations (Keresztes et al., 2017) may have promoted both more accurate responses during the frequency-learning task itself, and in participants' subsequent frequency judgments.

Frequency knowledge has been implicated in a wide range of cognitive processes (Hasher \& Zacks, 1984). For example, category and schema formation may be supported by knowledge of the frequency of different features or events, skilled reading can be facilitated by knowledge of the frequency of letter positions, which enable rapid word decoding, and individuals' judgments of the truth of facts or ideas are sensitive to the frequency with which they have previously encountered them (Hasher \& Zacks, 1984). Here, we suggest that the nature of frequency representations can also influence memory prioritization. Individuals who represented the lowand high-frequency items as more distinct from one another also demonstrated the greatest valuebased modulation of subsequent memory.

Developmental differences in explicit frequency knowledge did not explain all of the developmental variance we observed in value-guided memory. We also found that older participants demonstrated a greater effect of explicit frequency reports on memory, such that they demonstrated better memory for associations involving items they reported as being more frequent. This suggests that with increasing age, participants gained the ability to direct encoding resources toward items they believed were more frequent and therefore more valuable. These findings align with prior work that suggests that the use of value to modulate the implementation of strategic control processes during encoding may also continue to develop into early adulthood (Davidow et al., 2018).

It is worth noting that participants' accuracy in their frequency reports did not relate to subsequent memory for paired associates. Instead, participants' beliefs about the value of information predicted later memory. This finding is in line with those of previous studies that also observed a relation between participants' subjective beliefs about the importance of information and later memory (Friedman, McGillivray, Murayama, \& Castel, 2014; McGillivray \& Castel, 2017). That said, in our study, subjective and objective importance were closely related as the vast majority of participants were able to successfully differentiate the high- and low-frequency items (see Figure 2C). Though we did not observe a direct effect of the accuracy of participant's frequency reports on subsequent memory, maintaining representations that preserve the separation between low- and high-value information is likely critical to using prior experience to prioritize memory for information that is actually valuable. 


\section{LEARNED VALUE FACILITATES MEMORY OVER DEVELOPMENT}

Importantly, though we designed our task to reflect environments in which an item's frequency indicated the value of remembering its association, it is possible that our observed effects would have emerged even if participants did not implement a value-guided encoding strategy. Instead, the demand characteristics of the frequency-learning task, in which participants had to respond to the higher frequency items, and greater exposure to them may have facilitated stronger associative memory. Previous work has revealed that people are better at remembering pairs or triplets of items when they comprise constituents with stronger memory traces, like highfrequency relative to low-frequency words, or famous relative to non-famous faces, even when the associations themselves are equally novel (Chalmers \& Humphreys, 2010; Popov \& Reder, 2019; Reder, Liu, Keinath, \& Popov, 2015). Popov and Reder (2019) proposed that learning associations requires encoding each item individually as well as binding them together, which both impose demands on working memory. When memory traces for the individual items are stronger, there are more resources available in working memory to devote to learning the association itself, leading to better memory for associations involving well-known constituents. Thus, while we incentivized participants to remember associations involving high-frequency items, it is possible that frequency may have facilitated memory even in the absence of such an incentive structure. Rather than individuals using representations of item frequency as a proxy for information value to guide prioritized encoding, higher frequency alone may have generated stronger item memory traces, facilitating memory for associated information.

The pattern of results we observed in Experiment 1 cannot resolve these two competing explanations for how the statistical structure of the environment may influence memory across development. As we originally hypothesized, it may be the case that the emergence of better memory for associations involving high-frequency items across development was due largely to developmental improvements in the ability to explicitly represent item frequency and engage value-guided strategic control processes. Alternatively, it could be that greater exposure to the high-frequency items facilitated associative encoding in our older participants.

To better tease apart the different routes through which frequency may influence memory across development, we ran a second experiment. Here, we aimed to test the possibility that the effects of frequency condition on memory that we observed in Experiment 1 arose due to the increased exposure to high-frequency items during the frequency task rather than to strategic allocation of encoding resources due to the value manipulation. To de-confound these mechanistic explanations, we had a new group of participants complete a similar experiment where we removed the incentive for remembering associations involving high-frequency items such that participants could only earn one point for remembering each association, regardless of how many times they originally saw each item. While the structure of Experiment 1 was designed to capture how frequency might modulate information value in naturalistic environments, we purposefully broke this structural relation in Experiment 2, and instead explicitly instructed participants that all information would be equally rewarding to remember.

In Experiment 1, adults demonstrated the most robust effect of item frequency on associative memory. Given that any value-independent effect of item frequency on memory likely 


\section{LEARNED VALUE FACILITATES MEMORY OVER DEVELOPMENT}

also operated in Experiment 1, we reasoned that adults would also show the most robust effect of item frequency on memory even in the absence of the original value manipulation. Thus, in Experiment 2, we tested a new group of adult participants to determine the extent to which the statistics of the environment would influence memory in the absence of an explicitly instructed frequency-dependent reward structure.

\section{Method}

Preregistration. As in Experiment 1, we preregistered our study design and analysis plan (including specific models and inference criteria) on the Open Science Framework. We preregistered Experiment 2 methods after collecting and analyzing the data from Experiment 1, but prior to collecting or analyzing any data from Experiment 2.

Participants. To determine our sample size, we ran an additional analysis on our Experiment 1 data to ensure that our initial subset of 30 adults demonstrated a robust effect of frequency condition on memory. Specifically, we re-ran our mixed-effects model examining the influence of frequency condition, block, and their interaction on memory accuracy for the paired associates. Here, however, we included data only from the 30 adult subjects, and we removed our fixed effects of age and IQ from the model. We found that indeed, adults demonstrated significantly better memory for associations involving high-frequency items, $\chi^{2}(1)=14.26, p=$ .0002. Thus, we decided to use the same sample size for Experiment 2.

Thirty adult participants between the ages of 18.00 and 25.42 years (mean age $=21.98$

years; 16 females) took part in this experiment. Two additional participants were tested but excluded from all analyses due to a technical failure that caused some data files to be overwritten $(\mathrm{n}=1)$ and performing below-chance on the paired-associates task $(\mathrm{n}=1$; see 'Experimental Tasks' for details). Participants were recruited, consented, and compensated as in Experiment 1. Based on self-report, $43.3 \%$ of participants were Asian, 20\% were White, 16.7\% were mixed race, and 16.7\% were African American. Additionally, 16.7\% of the sample identified as Hispanic.

Experimental Tasks. As in Experiment 1, participants completed two sets of three tasks (Figure 1).

The first task, the frequency task, was identical to that used in Experiment 1 with one exception: Participants were instructed to try to keep track of how many of each kind of postcard they had, but they were not told that this information would be useful to them later.

The second task, the paired-associates task, was also largely identical to that used in Experiment 1 . Here, we altered only the instructions. Rather than reinforcing the relation between frequency and value, we explicitly informed participants that all information was equally useful to encode. Participants were told that they would need to remember the pairs of items and that they would win 1 point for each pair they remembered correctly. They were not instructed that they would earn more points by remembering the pairs associated with the high-frequency items.

The first two parts of the memory test were also identical to that from Experiment 1. Participants first identified the paired associate for each item once by selecting it from a large grid. They then reported how many times they believed they saw each item in the first task. They did 


\section{LEARNED VALUE FACILITATES MEMORY OVER DEVELOPMENT}

not, however, select the paired associate for their remaining items, meaning they stamped all the postcards they had 5 of and framed all the pictures they had 5 of one time only, such that participants would earn the same amount of points for remembering information associated with high- and low- frequency items.

Analysis approach. We followed the same analysis approach as in Experiment 1.

\section{Experiment 2 Results}

Confirmatory analyses. Paired-associate task. First, we confirmed that all participants performed above-chance on each block of the paired-associates task. To perform above chancelevel (50\%), participants had to correctly indicate on which side of the screen the stamp appeared on at least 12 out of 16 trials on each block. On average, participants responded correctly on $97.5 \%$ ( $\mathrm{SD}=5.1 \%$ ) of trials, with the lowest-performing participants still responding correctly on $75 \%$ of trials. As described in the methods, one additional participant was tested but excluded from all analyses for failing to reach this $75 \%$ threshold on one block of the paired-associates task.

Learning of item frequency. As in Experiment 1, we next examined how the accuracy and speed with which participants identified the repeating images in the frequency task changed as a function of block and the number of times the item had appeared in the task.

In general, participants were highly accurate in recognizing the new images, correctly withholding a response on $98.2 \%$ of trials $(S D=13.2 \%)$. A logistic mixed-effects model revealed no effect of experiment block on accuracy, $\chi^{2}(1)=.05, p=.82$. Participants were also highly accurate in recognizing images as repeated, correctly responding on $95.5 \%$ of trials $(S D=20.1 \%)$. We examined how participants' accuracy in recognizing the repeated images varied across block and the number of times each item had been presented (appearance count). We observed a main effect of appearance count on response accuracy, $\chi^{2}(1)=18.69, p<.0001$, indicating that participants responded more accurately as the number of item repetitions increased. A linear mixed-effects model also revealed that participants were faster to respond to the repeated items as their appearance counts increased, $F(1,27.23)=40.22, p<.0001$. No other effects reached significance $(p s>.06)$.

Data from the frequency-learning phase of our experiment suggest that, though participants were not explicitly told that learning item frequencies would be useful to them, they were able to recognize both the new and repeated images with a high degree of accuracy.

Explicit frequency reports. Next, we examined participants' explicit knowledge of the frequency of each item by examining the magnitude of participants' report error on each trial as a function of frequency condition and block. We observed a significant frequency condition $\mathrm{x}$ block interaction, $F(1,28.67)=5.00, p=.03$, such that participants responded more accurately to items in the 1 -frequency condition in the first block and more accurately to items in the 5-frequency condition in the second block. No other effects were statistically significant ( $p \mathrm{~s}>.67)$.

Frequency effects on memory. After confirming successful learning and memory for item frequencies, we examined the main question we sought to address with this second experiment: Did participants demonstrate better memory for associations involving higher-frequency items, 


\section{LEARNED VALUE FACILITATES MEMORY OVER DEVELOPMENT}

even when it would not be more rewarding to do so? To address this question, we examined how frequency condition, block, and their interactions influenced participants' memory accuracy with a logistic mixed-effects model. As in Experiment 1, we observed a main effect of block, $\chi^{2}(1)=$ $5.78, p=.02$, indicating that participants were better at remembering associations in the second block. Here, unlike in Experiment 1, we did not observe an effect of frequency condition on memory accuracy, $\chi^{2}(1)=.96, p=.33$, indicating that participants' memory across conditions did not significantly differ (Figure 5). This effect was not qualified by a block $\mathrm{x}$ frequency condition interaction $(p=.52)$. To quantify the strength of evidence in favor of the null hypothesis, we further conducted a Bayesian analysis of this effect, which suggested the data we observed were more than 18 times more likely to be observed under the null hypothesis relative to the alternative hypothesis (i.e. an effect of frequency condition; see Supplementary Analyses Table S33).

Relation between frequency reports and associative memory. We next examined whether there was a relation between participants' memory for item associations and their estimates of item frequency, as there was in Experiment 1. Here, since remembering associations involving highfrequency items would not lead to more reward, it is unlikely that participants would have purposefully prioritized memory for their associations. As such, a relation between associative memory and frequency reports would provide evidence in favor of stronger memory traces for individual items influencing both memory for their associations and beliefs about their original frequency. At the participant level, we did not observe a relation between frequency distance scores and memory benefit scores, $F(1,52.89)=2.46, p=.12$. At the item level, however, we did observe an effect of frequency report on memory, such that participants demonstrated higher memory accuracy for items they reported as being more frequent, $\chi^{2}(1)=8.54, p=.003$ (Figure 6).

Exploratory analyses: Comparison of Experiment 1 and Experiment 2. Frequencylearning. Finally, we more directly compared our memory findings from Experiment 1 and Experiment 2 by combining the adult data from both experiments. We first wanted to determine whether learning of item frequencies was similar across the two experiments. We re-ran our models examining online learning of item frequency and explicit frequency reports including experiment as an interacting fixed effect. Experiment did not significantly influence accuracy or reaction times during the frequency-learning task, nor was it related to frequency report error magnitude $(p s>.58)$. The only significant effect involving experiment that we observed across these models was a block $\mathrm{x}$ experiment $\mathrm{x}$ appearance count interaction effect on accuracy during the frequency learning task, $\chi^{2}(1)=4.80, p=.03$. Participants' accuracy in identifying items as old on third, fourth, and fifth appearances improved more across blocks in Experiment 1 relative to Experiment 2. Taken together, these results suggest that both online learning of item frequencies - and the transformation of this online learning into explicit representations - was largely similar across experiments.

Effects of item frequency on memory. Next, we examined whether item frequency differentially influenced memory across experiments. Though we observed a significant effect of frequency condition on associative memory accuracy in Experiment 1 but not in Experiment 2, that in and of itself does not indicate that memory performance differed significantly across 


\section{LEARNED VALUE FACILITATES MEMORY OVER DEVELOPMENT}

experiments. To test this question more directly, we re-ran our memory accuracy model on our combined adult data set, including experiment as an interacting fixed effect. As in Experiment 1, we found a significant effect of frequency condition on memory accuracy, such that participants demonstrated better associative memory for items in the 5-relative to the 1- frequency condition, $\chi^{2}(1)=12.58, p=.0004$. Critically however, this effect was qualified by a frequency condition $\mathrm{x}$ experiment interaction, $\chi^{2}(1)=8.55, p=.003$, such that participants demonstrated a greater effect of frequency condition in Experiment 1 relative to Experiment 2 (Figure 5).

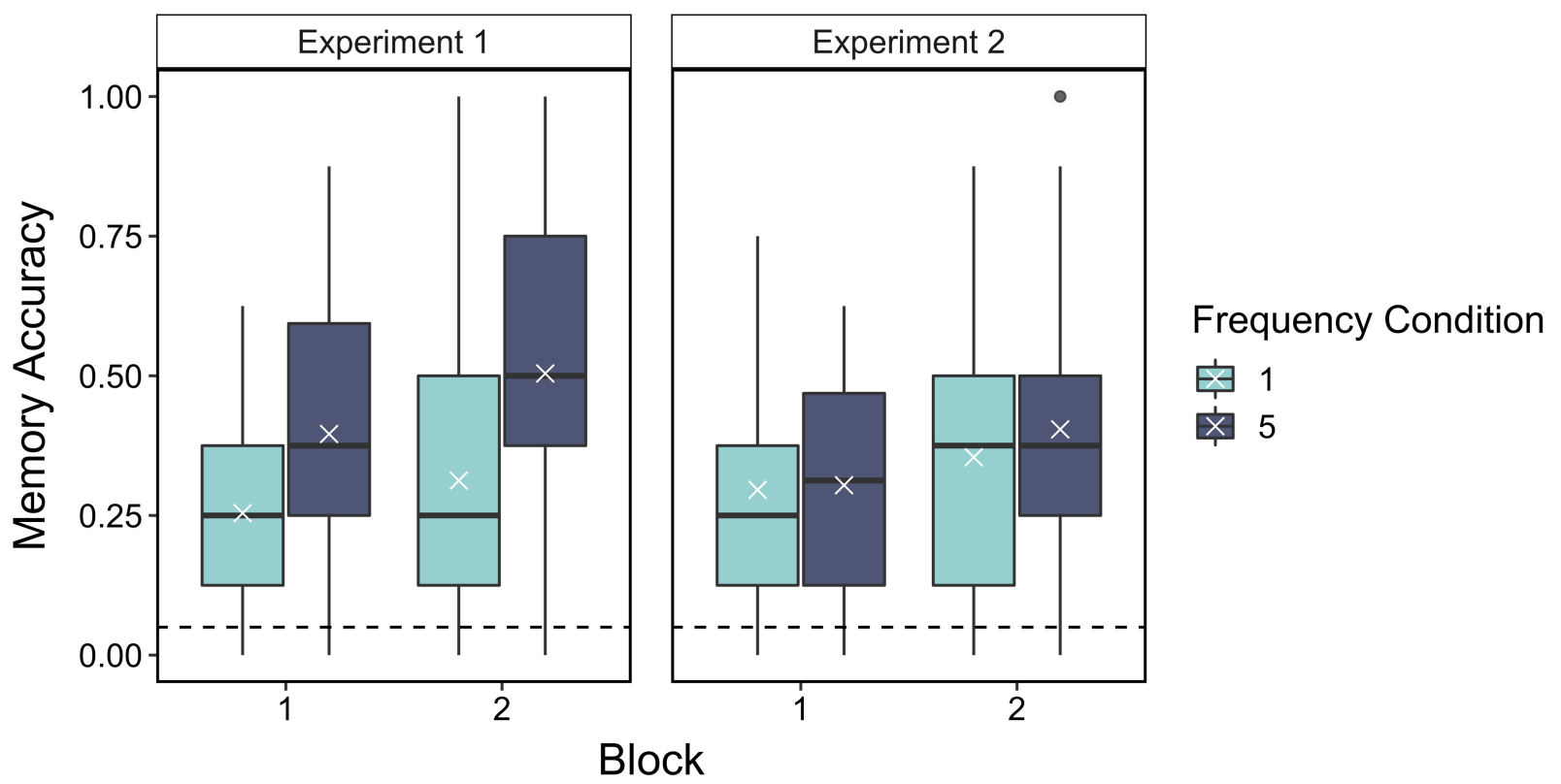

Figure 5. Adult participants demonstrated a significant effect of frequency condition on associative memory in Experiment $1(p=.0002)$, but not in Experiment $2(p=.33)$, in which frequency was not related to encoding value. The dashed line indicates chance-level performance.

To better disentangle the mechanisms that might support the relation between frequency reports and associative memory, we also re-ran our model examining the effects of frequency report on item memory. Experiment 2 suggested that people report items involved in more memorable associations as more frequent, even in the absence of a value manipulation. However, it may still be the case that in Experiment 1 participants purposefully prioritized memory for associations involving items they believed were more frequent. If this were true, we would expect the relation between frequency reports and memory to be stronger in Experiment 1 relative to Experiment 2. In line with this expectation, in addition to observing main effects of frequency report and block on memory ( $p \mathrm{~s}<.002)$, we observed an experiment $\mathrm{x}$ frequency report interaction, $\chi^{2}(1)=6.62, p=.01$, such that participants demonstrated a greater effect of frequency report on memory in Experiment 1 versus Experiment 2 (Figure 6). 


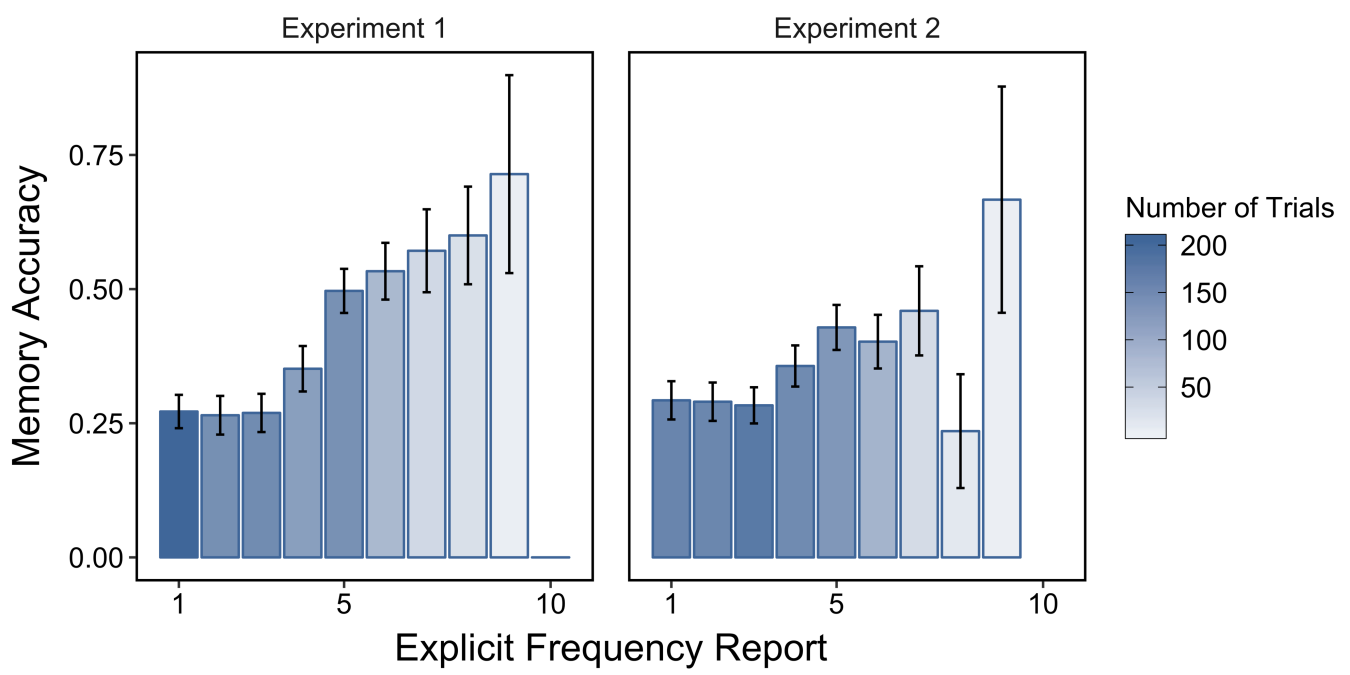

Figure 6. Adult participants' frequency reports were related to associative memory across both experiments. However, this effect was stronger in Experiment 1 relative to Experiment $2(p=.01)$.

\section{Experiment 2 Discussion}

In Experiment 2, we removed the relation between item frequency and the potential reward participants could earn by remembering associations involving each item. The goal of this manipulation was to de-confound the extent to which adults' better memory for associations involving high-frequency constituents was due to strategic modulation of encoding based on learned value signals versus increased prior exposure to the more frequent items. Whereas in Experiment 1, adult participants exhibited better memory for information related to high-frequency items, we did not observe such better memory performance for information related to highfrequency items in Experiment 2, when the incentive for remembering these associations was removed. Further, we confirmed that the effects of frequency condition on memory were significantly stronger in Experiment 1 than Experiment 2. This indicates that neither greater exposure to the higher frequency items, nor the demand characteristics of the frequency learning task, can explain the effects of frequency condition on memory that we previously observed in Experiment 1. Instead, the differences across the two experiments suggest that participants in Experiment 1 were using learned item frequencies as a signal of information value, and strategically modulating encoding accordingly.

Of course, we did not run the full developmental sample in Experiment 2. In Experiment 1 , adults demonstrated the strongest effect of frequency condition on memory. As such, we hypothesized that if an alternate, non-value-based mechanism - like prior exposure to more frequent items - facilitated enhanced memory in Experiment 1, it would have done so to the greatest extent in adults. However, it is still theoretically possible that the effects of frequency condition on memory that we observed in children and adolescents in Experiment 1 were driven to some extent by a different, non-value-based encoding mechanism. To the best of our knowledge, 


\section{LEARNED VALUE FACILITATES MEMORY OVER DEVELOPMENT}

no studies have examined how the influence of prior exposure on associative memory changes from childhood to adulthood. It could be the case that prior exposure to the more frequent stimuli facilitated memory in children and adolescents but not adults, whose memory performance was influenced by the link between frequency and value. To better tease apart the mechanisms underlying the influence of frequency on memory, future studies could examine how prior exposure to information facilitates associative memory across the lifespan. Still, the pattern of results we observed in Experiment 1 is highly consistent with other studies that have shown monotonic, age-related increases in the use of value to modulate cognitive control and attention more generally (Davidow et al., 2018; Insel et al., 2017; Störmer et al., 2014) and cognitive control over memory specifically (Castel et al., 2011), suggesting that our developmental effects were likely driven by a similar mechanism.

The results of Experiment 2 also help clarify the cognitive mechanisms that may underlie the relation between frequency reports and associative memory that we observed in Experiment 1. In our first experiment, we found that participants' explicit reports of item frequency predicted associative memory accuracy above and beyond the true underlying frequencies. Further, this relation strengthened with age. One interpretation of this relation is that participants used their explicit representations of item frequencies to modulate the extent to which they dedicated encoding resources to particular associations. However, we similarly found that explicit frequency reports predicted memory accuracy in Experiment 2, when there would have been no reason for participants to engage in such a top-down strategy. This suggests that a common mechanism may influence both associative memory and explicit frequency reports, leading to their correlation. For example, participants may have had stronger or more numerous memory traces for particular items, leading to both better associative encoding, and a subjective sense that the item was more frequent (Jonides \& Naveh-Benjamin, 1987). Alternatively, participants' memory accuracy may have influenced their representation of the frequency of each item. Scimeca and Badre (2012) proposed that successful retrieval of a memory indicates its utility within a particular context, and increases the likelihood that it will be needed again in the future. In this way, successful retrieval may have increased participants' estimates of an item's utility, biasing their belief about its initial frequency. Still, we did observe a significantly stronger relation between frequency reports and associative encoding in Experiment 1 relative to Experiment 2, suggesting that either or both of these effects likely operated in tandem with a top-down encoding strategy guided by participants' beliefs about the structure of their environment. Moreover, younger participants in Experiment 1 demonstrated a weaker relation between explicit frequency reports and subsequent associative memory. This suggests developmental modulation of at least one of these component processes. However, further work is needed to tease apart the developmental trajectories of the possible mechanisms that may have contributed to this relation.

\section{General Discussion}

Across two experiments, we examined how item frequency relates to associative memory, with a focus on how knowledge of information value modulates these effects across development. 


\section{LEARNED VALUE FACILITATES MEMORY OVER DEVELOPMENT}

In general, our findings aligned with our initial predictions: With increasing age, individuals became better both at deriving explicit knowledge of the frequency of items within their environment and at selectively encoding information associated with higher frequency items. However, differences in explicit frequency knowledge did not fully explain developmental differences in value-guided memory, suggesting that the development of dynamic control processes also contributes to increases in memory selectivity. These results highlight that deriving explicit knowledge of the structure of the environment from experience and flexibly adjusting the engagement of cognitive control processes comprise two mechanistic pathways that work in tandem across development to enable the environment itself to guide adaptive memory.

The flexible engagement of cognitive control over memory may have further relied on participants' recognition of the need to implement a selective encoding strategy. To mitigate developmental differences in participants' recognition that prioritizing memory for pairs involving high-frequency items would enable them to earn more points, we explicitly instructed this adaptive strategy with extensive explanation prior to the encoding task. Nevertheless, it is possible that younger participants demonstrated worse comprehension of the instructions and failed to implement a selective encoding strategy because they did not realize that doing so would be useful. A more likely possibility is that younger participants understood the instructed strategy but lacked the metacognitive awareness to recognize its importance. Previous studies have suggested both that memory monitoring improves from childhood to adulthood (Geurten, Catale, \& Meulemans, 2014; Ghetti, Mirandola, Angelini, Cornoldi, \& Ciaramelli, 2011; Grammer, Purtell, Coffman, \& Ornstein, 2011) and can promote the use of control strategies to selectively encode of high-value information (McGillivray \& Castel, 2017). Without strong metacognitive awareness of one's own memory capacity, children may have implemented poor memory strategies like trying to remember all the pairs. Additionally, they may not have accurately monitored the strength of their memory traces for previously encountered items, and failed to devote additional rehearsal time to weakly encoded, high-value pairs. Indeed, younger participants in Experiment 1 demonstrated not just poorer memory selectivity, but poorer memory performance overall. Though this may reflect generally weaker associative memory abilities (Shing et al., 2010), the poor performance of a large number of children is also likely due to a failure to selectively attend to and try to remember a more manageable subset of stimuli. Future work could more directly test how developmental change in metacognition supports value-guided memory selectivity by deriving richer measures of metacognitive monitoring through the use of experimental designs in which individuals have more control over encoding (Castel, Murayama, Friedman, McGillivray, \& Link, 2013; Ruggeri, Markant, Gureckis, Bretzke, \& Xu, 2019).

Our findings do not mean that frequency never facilitates memory in the absence of a particular reward structure. Indeed, many studies have shown a robust influence of prior item exposure on subsequent associative encoding (Chalmers \& Humphreys, 2003; Clark, 1992; Clark \& Shiffrin, 1992; Popov \& Reder, 2019; Reder, 2013; Reder, Liu, Keinath, \& Popov, 2015). Our results demonstrate, however, that drawing peoples' attention to a feature of many real-world environments - the link between item frequency and the future usefulness of related information 


\section{LEARNED VALUE FACILITATES MEMORY OVER DEVELOPMENT}

(J. R. Anderson \& Milson, 1989; J. R. Anderson \& Schooler, 1991) — boosts the influence of frequency on memory, at least in early adulthood. Engaging a value-based strategy may be particularly effective in contexts like ours, where item memory traces may not be as strong as they are for very well-known constituents that are already deeply embedded in extensive semantic networks, like famous faces or highly frequent words.

Further, as we noted in our introduction, frequency is only one naturalistic signal of the importance of information. Individuals can also prioritize information in memory by relying on their own curiosity or emotional responses to information (Adelman \& Estes, 2013; Kang et al., 2009), their prior knowledge (Brod et al., 2013), or other signals related to the statistics of the environment, like how novel an item is (Tulving \& Kroll, 1995), or how recently an item has been encountered (J. R. Anderson \& Milson, 1989). In real-world environments, these different value signals likely interact with each other. Additionally, the extent to which they predict the future utility of remembering information may differ across contexts. For example, in some environments, it may be more useful to remember information associated with rare or unusual items, whereas in others, remembering information associated with more frequent items may ultimately lead to more reward. As we demonstrated here, individuals' ability to use these signals to prioritize memory may change across development. Similarly, individuals' ability to adjust the extent to which they weight these different value signals depending on their validity within a given context may also change with age. In natural environments, learning to adapt memory to the statistics of the environment may also recruit meta-learning processes that help individuals arbitrate between different learned value cues (Griffiths et al., 2019; Lieder \& Griffiths, 2017). Future studies could vary the validity of different types of value cues and measure the extent to which individuals across a wide age range adjust their memory strategies accordingly.

Future work should also examine the neural mechanisms that underlie developmental change in the influence of learned value on strategic encoding. Neural data could potentially help dissociate improvement in individuals' recognition of an item's value from the strategic use of that value signal to control encoding processes. We expect value recognition to be reflected in interactions between medial temporal areas involved in retrieving representations of item frequency (Davachi, Mitchell, \& Wagner, 2003; Ghetti \& Bunge, 2012) and midbrain regions associated with reward anticipation (Adcock et al,, 2006; Wolosin, Zeithamova, \& Preston, 2012), whereas strategic control of encoding may rely on interactions between those midbrain regions and prefrontal cortical areas implicated in cognitive control (Church, Bunge, Petersen, \& Schlaggar, 2016; Insel et al., 2017; Miller \& Cohen, 2001). Specifically, age-related increases in connectivity between the striatum and the ventrolateral prefrontal cortex may support the selective engagement of control processes in response to value (Davidow et al., 2018; Insel et al., 2017; Shing, Werkle-Bergner, Li, \& Lindenberger, 2008).

The ability to use prior learning or inferred value signals to guide memory is particularly important in real-world environments because information value often changes across contexts. Children and adolescents in particular face sharp discontinuities in their environments as their social and physical worlds transform. While these discontinuities may change the utility of specific 


\section{LEARNED VALUE FACILITATES MEMORY OVER DEVELOPMENT}

information, the general strategy of encoding information associated with frequently encountered contexts often remains robust even as the contexts themselves change. A child who frequently visits the playground might benefit from remembering information about it - like the best hiding spots for hide-and-seek - but the value of that knowledge will decrease as she grows up and her visits become less frequent. That same child, however, will continue to benefit from prioritizing information associated with frequent contexts throughout her life — the best playground hiding spots may be replaced by the hours of her favorite store in the mall, the most comfortable chairs in her college library, the tastiest cocktail at her neighborhood bar. In this way, individuals' gradual developmental increases in the ability to use learned environmental regularities to dynamically prioritize memory for useful information support increasingly adaptive behavior in an everchanging world.

\section{Acknowledgments}

We thank Daryl Ocampo, Jamie Greer, and Nora Keathley for assistance with data collection, and the National Science Foundation (CAREER grant 1654393 to C.A.H.), Jacobs Foundation (Early Career Fellowship to C.A.H), Department of Defense (NDSEG Fellowship to K.N.), and New York University (DURF grant to E.P.) for research support.

\section{Author Contributions}

K.N. and C.A.H. designed the study. K.N. and E.P. collected and analyzed the data under the supervision of C.A.H. K.N. and C.A.H. wrote the manuscript. All authors approved the final version of the manuscript for submission.

\section{Author Note}

The data, task code, analysis code, and preregistrations for both experiments described in the manuscript can be accessed on the Open Science Framework: https://osf.io/h9ncs/

Some of the data and ideas in the manuscript were presented at the 2018 meetings of the Society for Neuroeconomics, the Flux Society for Developmental Cognitive Neuroscience, and the Social and Affective Neuroscience Society, as well as at the UC Irvine International Conference on Learning and Memory.

(C) 2020, American Psychological Association. This paper is not the copy of record and may not exactly replicate the final, authoritative version of the article. Please do not copy or cite without authors' permission. The final article will be available, upon publication, via its DOI: $10.1037 /$ xge0000753 


\section{References}

Adelman, J. S., \& Estes, Z. (2013). Emotion and memory: a recognition advantage for positive and negative words independent of arousal. Cognition, 129(3), 530-535. doi:10.1016/j.cognition.2013.08.014

Amso, D., \& Davidow, J. (2012). The development of implicit learning from infancy to adulthood: item frequencies, relations, and cognitive flexibility. Developmental Psychobiology, 54(6), 664-673. doi:10.1002/dev.20587

Anderson, J. R. (1991). Is human cognition adaptive? Behavioral and Brain Sciences, 14(3), 471-485. doi:10.1017/S0140525X00070801

Anderson, J. R., \& Milson, R. (1989). Human Memory: An Adaptive Perspective. Psychological Review, 96(4), 703-719. doi:10.1037/0033-295X.96.4.703

Anderson, J. R., \& Schooler, L. J. (1991). Reflections of the environment in memory. Psychological Science, 2(6), 396-408. doi:10.1111/j.1467-9280.1991.tb00174.x

Anderson, R. B., Tweney, R. D., Rivardo, M., \& Duncan, S. (1997). Need probability affects retention: A direct demonstration. Memory \& Cognition, 12(6), 867-872. doi: 10.3758/BF03211331

Barr, D. J., Levy, R., Scheepers, C., \& Tily, H. J. (2013). Random effects structure for confirmatory hypothesis testing: Keep it maximal. Journal of Memory and Language, 68(3), 255-278. doi:1016/j.jml.2012.11.001

Bransford, J. D., \& Johnson, M. K. (1972). Contextual prerequisites for understanding: Some investigations of comprehension and recall. Journal of Verbal Learning and Verbal Behavior, 11(6), 717-726. doi:10.1016/S0022-5371(72)80006-9

Brod, G., \& Shing, Y. L. (2019). A boon and a bane: Comparing the effects of prior knowledge on memory across the lifespan. Developmental Psychology, 55(6), 1326-1337. doi: $10.1037 / \operatorname{dev} 0000712$

Brod, G., Werkle-Bergner, M., \& Shing, Y. L. (2013). The influence of prior knowledge on memory: a developmental cognitive neuroscience perspective. Frontiers in Behavioral Neuroscience, 7. doi:10.3389/fnbeh.2013.00139

Bürkner, P. (2017). brms: An R Package for Bayesian Multilevel Models Using Stan. Journal of Statistical Software, 80(1), 1-28. doi:10.18637/jss.v080.i01.

Bürkner, P. (2018). Advanced Bayesian Multilevel Modeling with the R Package brms. The $R$ Journal, 10(1), 395-411. doi:10.32614/RJ-2018-017.

Castel, A. D., Humphreys, K. L., Lee, S. S., Galván, A., Balota, D. A., \& McCabe, D. P. (2011). The development of memory efficiency and value-directed remembering across the life span: A cross-sectional study of memory and selectivity. Developmental Psychology, 47(6), 15531564. doi:10.1037/a0025623

Castel, A. D., Murayama, K., Friedman, M. C., McGillivray, S., \& Link, I. (2013). Selecting valuable information to remember: Age-related differences and similarities in self-regulated learning. Psychology and Aging, 28(1), 232-242. doi:10.1037/a0030678 


\section{LEARNED VALUE FACILITATES MEMORY OVER DEVELOPMENT}

Chalmers, K., \& Humphreys, M. (2010). Experimental manipulation of prior experience: Effects on item and associative recognition. Memory, 11(3), 233-246.

doi:10.1080/09658210244000009

Church, J. A., Bunge, S. A., Petersen, S. E., \& Schlaggar, B. L. (2016). Preparatory Engagement of Cognitive Control Networks Increases Late in Childhood. Cerebral Cortex, 27(3), 21392153. doi:10.1093/cercor/bhw046

Craik, F. I. M., \& Watkins, M. J. (1973). The Role of Rehearsal in Short-Term Memory. Journal of Verbal Learning and Verbal Behavior, 12, 599-607. doi:10.1016/S0022-5371(73)80039-8

Davachi, L., Mitchell, J. P., \& Wagner, A. D. (2003). Multiple routes to memory: distinct medial temporal lobe processes build item and source memories. Proceedings of the National Academy of Sciences, 100(4), 2157-2162. doi:1073/pnas.0337195100

Davidow, J. Y., Insel, C., \& Somerville, L. H. (2018). Adolescent Development of Value-Guided Goal Pursuit. Trends in Cognitive Sciences, 1-12. http://doi.org/10.1016/j.tics.2018.05.003

Decker, J. H., Lourenco, F. S., Doll, B. B., \& Hartley, C. A. (2015). Experiential reward learning outweighs instruction prior to adulthood. Cognitive, Affective, \& Behavioral Neuroscience, 15(2), 310-320. doi:10.3758/s13415-014-0332-5

Decker, J. H., Otto, A. R., Daw, N. D., \& Hartley, C. A. (2016). From Creatures of Habit to Goal-Directed Learners. Psychological Science, 27(6), 848-858. doi:10.1177/0956797616639301

Ellis, N. R., Palmer, R. L., \& Reeves, C. L. (1988). Developmental and intellectual differences in frequency processing. Developmental Psychology, 24(1), 38-45. doi:10.1037/00121649.24.1.38

Fandakova, Y., \& Gruber, M. (2019). Curiosity and surprise enhance memory differently in adolescents than in children. PsyArxiv. doi:10.31234/osf.io/s36e5.

Finn, A. S., Kalra, P. B., Goetz, C., Leonard, J. A., Sheridan, M. A., \& Gabrieli, J. D. E. (2016). Developmental dissociation between the maturation of procedural memory and declarative memory, Journal of Experimental Child Psychology, 142, 212-220.

doi:10.1016/j.jecp.2015.09.027

Friedman, M. C., McGillivray, S., Murayama, K., \& Castel, A. D. (2014). Memory for medication side effects in younger and older adults: The role of subjective and objective importance. Memory \& Cognition, 43(2), 206-215. doi:10.3758/s13421-014-0476-0

Geurten, M., Catale, C., \& Meulemans, T. (2014). When Children“s Knowledge of Memory Improves Children"s Performance in Memory. Applied Cognitive Psychology, 29(2), 244252. doi:10.1002/acp.3102

Ghetti, S., \& Bunge, S. A. (2012). Neural changes underlying the development of episodic memory during middle childhood. Developmental Cognitive Neuroscience, 1-15. doi:10.1016/j.den.2012.05.002

Ghetti, S., Mirandola, C., Angelini, L., Cornoldi, C., \& Ciaramelli, E. (2011). Development of Subjective Recollection: Understanding of and Introspection on Memory States. Child Development, 82(6), 1954-1969. doi: 10.1111/j.1467-8624.2011.01645.x 


\section{LEARNED VALUE FACILITATES MEMORY OVER DEVELOPMENT}

Grammer, J. K., Purtell, K. M., Coffman, J. L., \& Ornstein, P. A. (2011). Relations between children's metamemory and strategic performance: Time-varying covariates in early elementary school. Journal of Experimental Child Psychology, 108(1), 139-155. doi:10.1016/j.jecp.2010.08.001

Griffiths, T. L., Callaway, F., Chang, M. B., Grant, E., Krueger, P. M., \& Lieder, F. (2019). Doing more with less: meta-reasoning and meta-learning in humans and machines. Current Opinion in Behavioral Science, 29, 24-30. doi:10.1016/j.cobeha.2019.01.005

Hanten, G., Li, X., Chapman, S. B., Swank, P., Gamino, J., Roberson, G., \& Levin, H. S. (2007). Development of Verbal Selective Learning. Developmental Neuropsychology, 32(1), 585596. doi:10.1080/87565640701361112

Hasher, L., \& Chromiak, W. (1977). The processing of frequency information: An automatic mechanism? Journal of Verbal Learning \& Verbal Behavior, 16(2), 173184. doi:10.1016/S0022-5371(77)80045-5

Insel, C., Kastman, E. K., Glenn, C. R., \& Somerville, L. H. (2017). Development of corticostriatal connectivity constrains goal-directed behavior during adolescence. Nature Communications, 1-10. doi:10.1038/s41467-017-01369-8

Jonides, J., \& Naveh-Benjamin, M. (1987). Estimating frequency of occurrence. Journal of Experimental Psychology: Learning, Memory, and Cognition, 13(2), 230-240. doi: 10.1037/0278-7393.13.2.230

Kang, M. J., Hsu, M., Krajbich, I. M., Loewenstein, G., McClure, S. M., Wang, J. T.-Y., \& Camerer, C. F. (2009). The wick in the candle of learning: epistemic curiosity activates reward circuitry and enhances memory. Psychological Science, 20(8), 963-973. doi:10.1111/j.1467-9280.2009.02402.x

Keresztes, A., Bender, A. R., Bodammer, N. C., Lindenberger, U., Shing, Y. L., \& WerkleBergner, M. (2017). Hippocampal maturity promotes memory distinctiveness in childhood and adolescence. Proceedings of the National Academy of Sciences of the United States of America, 114(34), 9212-9217. doi:10.1073/pnas.1710654114

Hasher, L., \& Zacks, R. T. (1984). Automatic processing of fundamental information: the case of frequency of occurrence. The American Psychologist, 39(12), 1372-1388. doi:10.1037//0003-066x.39.12.1372

Lieder, F., \& Griffiths, T. L. (2017). Strategy selection as rational metareasoning. Psychological Review, 124(6), 762-794. doi:10.1037/rev0000075

Marvin, C. B., \& Shohamy, D. (2016). Curiosity and reward: Valence predicts choice and information prediction errors enhance learning. Journal of Experimental Psychology: General, 145(3), 266-272. doi:10.1037/xge0000140

McGillivray, S., \& Castel, A. D. (2017). Older and Younger Adults' Strategic Control of Metacognitive Monitoring: The Role of Consequences, Task Experience, and Prior Knowledge. Experimental Aging Research, 43(3), 233-256.

doi:10.1080/0361073X.2017.1298956 


\section{LEARNED VALUE FACILITATES MEMORY OVER DEVELOPMENT}

McGillivray, S., Murayama, K., \& Castel, A. D. (2015). Thirst for knowledge: The effects of curiosity and interest on memory in younger and older adults. Psychology and Aging, 30(4), 835-841. doi:10.1037/a0039801

Meulemans, T., \& Van der Linden, M. (1998). Implicit Sequence Learning in Children. Journal of Experimental Child Psychology, 69, 199-221. doi:10.1006/jecp.1998.2442

Miller, E. K., \& Cohen, J. D. (2001). An integrative theory of prefrontal cortex function. Annual Review of Neuroscience, 24(1), 167-202. doi:10.1146/annurev.neuro.24.1.167

Nussenbaum, K., Prentis, E., \& Hartley, C. A. (2019, August 8). Memory's reflection of learned information value increases across development. Retrieved from osf.io/h9ncs.

Pachur, T., Schooler, L. J., \& Stevens, J. R. (2014). We'll Meet Again: Revealing Distributional and Temporal Patterns of Social Contact. Plos One, 9(1), e86081-10. doi:10.1371/journal.pone.0086081

Popov, V., \& Reder, L. M. (2020). Frequency effects on memory: A resource-limited theory. Psychological Review, 127(1), 1-46. doi:10.1037/rev0000161

Potter, T. C. S., Bryce, N. V., \& Hartley, C. A. (2016). Cognitive components underpinning the development of model-based learning. Developmental Cognitive Neuroscience, 1-9. doi:10.1016/j.den.2016.10.005

R Core Team. (2013). R: A language and environment for statistical computing.

Reber, A. S., Walkenfeld, F. F., \& Hernstadt, R. (1991). Implicit and explicit learning: Individual differences and IQ. Journal of Experimental Psychology: Learning, Memory, and Cognition, 17(5), 888-896. doi:10.1037//0278-7393.17.5.888

Reder, L. M., Liu, X. L., Keinath, A., \& Popov, V. (2015). Building knowledge requires bricks, not sand: The critical role of familiar constituents in learning. Psychonomic Bulletin \& Review, 23(1), 271-277. doi:10.3758/s13423-015-0889-1

Ruggeri, A., Markant, D. B., Gureckis, T. M., Bretzke, M., \& Xu, F. (2019). Memory enhancements from active control of learning emerge across development. Cognition, 186, 82-94. doi:10.1016/j.cognition.2019.01.010

Saffran, J. R., \& Kirkham, N. Z. (2017). Infant Statistical Learning. Annual Review of Psychology, 69(1), 181-203. doi:10.1146/annurev-psych-122216-011805

Schlichting, M. L., \& Preston, A. R. (2015). Memory integration: neural mechanisms and implications for behavior. Current Opinion in Behavioral Sciences, 1, 1-8. doi:10.1016/j.cobeha.2014.07.005

Schlichting, M. L., Guarino, K. F., Schapiro, A. C., Turk-Browne, N. B., \& Preston, A. R. (2017). Hippocampal Structure Predicts Statistical Learning and Associative Inference Abilities during Development. Journal of Cognitive Neuroscience, 29(1), 37-51. doi:10.1162/jocn_a_01028

Schlüter, H., Hackländer, R. P., \& Bermeitinger, C. (2019). Emotional oddball: A review on memory effects. Psychonomic Bulletin \& Review, 26(5), 1472-1502. doi:10.3758/s13423019-01658-x 


\section{LEARNED VALUE FACILITATES MEMORY OVER DEVELOPMENT}

Scimeca, J. M., \& Badre, D. (2012). Striatal Contributions to Declarative Memory Retrieval. Neuron, 75(3), 380-392. doi:10.1016/j.neuron.2012.07.014

Shing, Y. L., Werkle-Bergner, M., Brehmer, Y., MÃ/4ler, V., Li, S.-C., \& Lindenberger, U. (2010). Episodic memory across the lifespan: The contributions of associative and strategic components. Neuroscience and Biobehavioral Reviews, 34(7), 1080-1091.

doi:10.1016/j.neubiorev.2009.11.002

Shing, Y. L., Werkle-Bergner, M., Li, S.-C., \& Lindenberger, U. (2008). Associative and strategic components of episodic memory: A life-span dissociation. Journal of Experimental Psychology: General, 137(3), 495-513. doi:10.1037/0096-3445.137.3.495

Shohamy, D., \& Adcock, R. A. (2010). Dopamine and adaptive memory. Trends in Cognitive Sciences, 14(10), 464-472. doi:10.1016/j.tics.2010.08.002

Singmann, H., Bolker, B., Westfall, J., Aust, F., Hojsgaard, S., Fox, J., et al. (2016). afex: Analysis of Factorial Experiments. Comprehensive R Archive Network (CRAN). Retrieved from https://cran.r-project.org/web/packages/afex/index.html

Störmer, V., Eppinger, B., \& Li, S.-C. (2014). Reward speeds up and increases consistency of visual selective attention: a lifespan comparison. Cognitive, Affective, \& Behavioral Neuroscience, 14(2), 659-671. doi:10.3758/s13415-014-0273-z

Tulving, E., \& Kroll, N. (1995). Novelty assessment in the brain and long-term memory encoding. Psychonomic Bulletin \& Review, 2(3), 387-390. doi:3758/BF03210977

Uncapher, M. R., \& Rugg, M. D. (2009). Selecting for Memory? The Influence of Selective Attention on the Mnemonic Binding of Contextual Information. Journal of Neuroscience, 29(25), 8270-8279. doi:10.1523/JNEUROSCI.1043-09.2009

Unger, K., Ackerman, L., Chatham, C. H., Amso, D., \& Badre, D. (2016). Working memory gating mechanisms explain developmental change in rule-guided behavior. Cognition, 155(C), 8-22. doi:10.1016/j.cognition.2016.05.020

Wagenmakers, E. (2007). A practical solution to the pervasive problems of $\mathrm{p}$ values. Psychonomic Bulletin and Review, 14(5), 779 - 804. doi: 10.3758/bf03194105.

Wechsler, D. (2011). Wechsler Abbreviated Scale of Intelligence (WASI-II) -- Second Edition. San Antonio, TX: NCS Pearson. doi:10.1037/t15171-000

Wilhelm, I., Rose, M., Imhof, K. I., Rasch, B., Büchel, C., \& Born, J. (2013). The sleeping child outplays the adult's capacity to convert implicit into explicit knowledge. Nature Neuroscience, 16(4), 391-393. doi:10.1038/nn.3343

Wittmann, B. C., Schott, B. H., Guderian, S., Frey, J. U., Heinze, H.-J., \& Düzel, E. (2005). Reward-Related fMRI Activation of Dopaminergic Midbrain Is Associated with Enhanced Hippocampus- Dependent Long-Term Memory Formation. Neuron, 45(3), 459-467. doi:10.1016/j.neuron.2005.01.010 